\title{
Quantum systems in and out of equilibrium
}

\section{Fundamentals, dynamics, and applications}

\author{
Pedro L. Garrido ${ }^{\mathrm{a}}$, Pablo Hurtado ${ }^{\mathrm{b}}$, Daniel Manzano ${ }^{\mathrm{c}}$, and Francisco de los Santos ${ }^{\mathrm{d}}$ \\ Department of Electromagnetism and Physics of Matter and Institute Carlos I for \\ Theoretical and Computational Physics, University of Granada, 18010 Granada, Spain
}

Received 18 May 2018 / Received in final form 7 June 2018

Published online 28 September 2018

This special issue originated at the 14th Granada Seminar on Quantum Systems in and out of Equilibrium: Fundamentals, dynamics and applications, which took place in 2017, from June 20 to June 23 in Granada, Spain. This edition was sponsored by the University of Granada through the Department of Electromagnetism and Physics of Matter and the Institute Carlos I for Theoretical and Computational Physics, the Spanish Ministry of Economy, Industry and Competitiveness, and the European Physical Society.

There were in this edition a total of 57 lectures and 27 poster contributions covering quantum aspects of thermalization, quantum transport, quantum effects in condensed matter, biology, quantum computation, open quantum systems, quantum fluctuations and large deviations, and quantum thermodynamics. Poster presenters were given the opportunity to explain their posters to the full audience in short, three-minute presentations limited to two slides. Near one hundred participants came from 27 countries (Spain contributed with 24\%; the rest of Europe including Russia, Ukraine, and Israel with 48\%; the Americas with 9\%; Asia with 18\%; and Africa with $1 \%)$.

This volume is a collection of fifteen peer-reviewed original contributions and two minireviews from conference lecturers on a varied range of fields related to the Seminar topics. A sizable number of contributions cover the field of quantum thermodynamics from quite different perspectives. In particular, Vinjanampathy and coworkers [1] report on a variational control algorithm to speed up thermalization in open quantum systems; Gallavotti [2] addresses the problem of finite thermostats in classical and quantum nonequilibrium; Barra and Lledo [3] study the thermodynamics of a quantum system interacting with different baths in the repeated interaction framework; del Campo et al. [4] investigate the decay of a thermofield-double state in chaotic quantum systems; Prior and coworkers [5] propose studying the properties and behaviour of organic solar cells through the modification of photocurrent generation when an external magnetic field is applied; Sugino and Padmanabhan [6] use modified Motzkin walks to study quantum phase transitions in a frustration-free spin chain; and Manzano [7] reports on entropy production and fluctuations in a Maxwell's refrigerator with squeezing.

\footnotetext{
a e-mail: garrido@onsager.ugr.es

b e-mail: phurtado@onsager.ugr.es

c e-mail: manzano@onsager.ugr.es

d e-mail: fdlsant@ugr.es
} 
A second large group of contributions deal with open quantum systems. Cabrera and coworkers [8] investigate non-ergodic states induced by impurity levels in the XY model; Poletti and coworkers [9] study the response of a particle current to dissipative dephasing in an interacting, few body fermionic lattice system; and Shirai et al. [10] study the long-time asymptotic states of periodically driven quantum systems coupled to a thermal bath by the use of a floquet state.

A third group covers rather different topics, including information entropies, atomic physics or quantum computation. Zozor and coworkers [11] study generalized entropies in quantum and classical statistical theories; Toranzo and coworkers [12] determine the exact values of the Rényi uncertainty measures of D-dimensional harmonic systems; Ramsak et al. [13] consider exactly solvable manipulation of spinorbit qubits confined in a moving harmonic trap and in the presence of the time dependent Rashba interaction; lastly, Kastner and Uhrich [14] generalise a former result to prove that special observables exist for which measurement backaction is of no concern when measuring temporal correlations.

Turning to the minireviews, Spagnolo et al. [15] review on noise induced phenomena in quantum metastable systems, while Buca and Prosen [16] discuss recent progress in the analytical study of certain current-carrying nonequilibrium steady states.

Finally, on the experimental side, Rodríguez and coworkers [17] report on the progress towards the implementation of a double trap system for the coupling of ion clouds stored in different traps that can be used either as Penning or Paul traps.

We wish to express here our gratitude to the authors and reviewers, and to all those who have collaborated in making the 14th Granada Seminar a success. We are grateful to the Local Organizing Committee, José Manuel Martín, Irene Adroher, Nicolás Tizón, and Paula Villa. Financial support from Spanish projects FIS2013-43201-P and FIS201784256-P (MINECO), and Junta de Andalucía and EU Project TAHUB/II-148 (Program ANDALUCÍA TALENT-HUB 291780) is acknowledged.

\section{References}

1. N. Suri, F.C. Binder, B. Muralidharan, S. Vinjanampathy, Eur. Phys. J. Special Topics 227, 203 (2018)

2. G. Gallavotti, Eur. Phys. J. Special Topics 227, 217 (2018)

3. F. Barra, C. Lledo, Eur. Phys. J. Special Topics 227, 231 (2018)

4. A. del Campo, J. Molina-Vilaplana, L.F. Santos, J. Sonner, Eur. Phys. J. Special Topics 227, 247 (2018)

5. S. Oviedo-Casado, A. Urbina, J. Prior, Eur. Phys. J. Special Topics 227, 259 (2018)

6. F. Sugino, P. Padmanabhan, Eur. Phys. J. Special Topics 227, 269 (2018)

7. G. Manzano, Eur. Phys. J. Special Topics 227, 285 (2018)

8. A.O. García Rodríguez, G.G. Cabrera, Eur. Phys. J. Special Topics 227, 301 (2018)

9. K. Choo, U. Bissbort, D. Poletti, Eur. Phys. J. Special Topics 227, 313 (2018)

10. T. Shirai, T. Mori, S. Miyashita, Eur. Phys. J. Special Topics 227, 323 (2018)

11. M. Portesi, F. Holik, P.W. Lamberti, G.M. Bosyk, G. Bellomo, S. Zozor, Eur. Phys. J. Special Topics 227, 335 (2018)

12. D. Puertas-Centeno, I.V. Toranzo, J.S. Dehesa, Eur. Phys. J. Special Topics 227, 345 (2018)

13. A. Ramsak, T. Cadez, A. Kregar, L. Ulcakar, Eur. Phys. J. Special Topics 227, 353 (2018)

14. M. Kastner, P. Uhrich, Eur. Phys. J. Special Topics 227, 365 (2018)

15. B. Spagnolo, A. Carollo, D. Valenti, Eur. Phys. J. Special Topics 227, 379 (2018)

16. B. Buca, T. Prosen, Eur. Phys. J. Special Topics 227, 421 (2018)

17. R.A. Rica, F. Domínguez, M.J. Gutiérrez, J. Bañuelos, J.J. del Pozo, D. Rodríguez, Eur. Phys. J. Special Topics 227, 445 (2018) 